\title{
Early Experience, Setup, Learning Curve, Benefits, and Complications Associated with Exoscope and Three-Dimensional 4K Hybrid Digital Visualizations in Minimally Invasive Spine Surgery
}

\author{
Mohd Hisam Muhamad Ariffin ${ }^{1}$, Kamalnizat Ibrahim ${ }^{1}$, Azmi Baharudin ${ }^{1}$, Azmi Mohd Tamil ${ }^{2}$ \\ ${ }^{1}$ Department of Orthopaedics, Faculty of Medicine, UKM Medical Centre, Kuala Lumpur, Malaysia \\ ${ }^{2}$ Department of Public Health, Faculty of Medicine, UKM Medical Centre, Kuala Lumpur, Malaysia
}

Study Design: Prospective observational study (n=74).

Purpose: To evaluate the learning curve for exoscope and three-dimensional (3D) 4K hybrid visualization in terms of operating time, advantages, disadvantages, and surgical complications in tubular-access minimally invasive spine surgery (MISS) and to assess surgeon satisfaction with image quality, ergonomics, and ability to perform target site treatment.

Overview of Literature: Working through tubular retractors poses a challenge. The extreme angulations during microsurgical decompression, especially contralateral decompression, require surgeons to work non-ergonomically. An exoscope allows surgeons to work ergonomically and independently of the microscope oculars as visualizations are now provided by large 3D 4K monitors. However, the value and efficacy of solely depending on an exoscope and 3D 4K monitors during microsurgical work are still unknown. Methods: Seventy-four patients (99 levels) underwent trans-tubular MISS between March 2018 and January 2019. Five patients were excluded: one had pyogenic discitis, two had revisions, and two were trans-tubular transoral. In total, we analyzed 69 for operating time, blood loss, and complications. The learning curve graph was plotted using the surgical time for each procedure. Surgeons were asked to rate their satisfaction with image quality, ability to maintain ergonomic posture, and efficient target site treatment.

Results: For tubular microdiscectomy, the operating time plateaued after six cases, and for tubular decompression and minimally invasive transforaminal lumbar interbody fusion, the operating time plateaued after nine cases. Mean operating time was significantly reduced after the plateau. Complications included four cases of dural tear. All patients improved symptomatically, and there were no postoperative neurological deficits.

Conclusions: Use of the exoscope has a short learning curve. Surgeons benefit from improved ergonomic posture during surgery, and resident teaching appears to be good. The only drawback is the need to rearrange the operating table setup. Complications were comparable to those when using the surgical microscope. An exoscope with hybrid digital visualization provides excellent visualization, depth perception, clarity, and precision target site treatment.

Keywords: Exoscope; Minimally invasive spine surgery

Received Mar 12, 2019; Revised May 5, 2019; Accepted May 8, 2019

Corresponding author: Mohd Hisam Muhamad Ariffin

Department of Orthopaedics, Faculty of Medicine, UKM Medical Centre, Cheras, Kuala Lumpur, Malaysia

Tel: +60-194518686, Fax: +60-391456674, E-mail: hesam8791@yahoo.com 


\section{Introduction}

The surgical microscope has revolutionized spinal microsurgery. It allows the simultaneous visualization and illumination of the surgical field and has improved patient outcomes. Certain microsurgical procedures like microdiscectomy have become a gold standard treatment [1-3]. Further development in minimally invasive spine surgery (MISS) introduced the tubular retractor system along with angled and bayonetted instruments such that the line of sight through a tubular retractor is always unobstructed. Patients have less postoperative pain, have shorter hospital stays, and return to work more rapidly [4,5]. In addition, analgesic consumption was significantly reduced in patients operated via the transmuscular route compared to the subperiosteal approach [6]. This is likely due to less muscle damage and lower muscle pressure from the retractor $[7,8]$.

However, working through a tubular retractor with a surgical microscope constrains surgeons. The extreme microscope positional angles required for successful decompression, especially the contralateral aspect, compromises the surgeons' neutral posture, forcing them to adopt a non-ergonomic posture, often for extended periods of time. When positioning the microscope at extreme angles, surgeons will typically extend their arms to reach the surgical field, flex their hips and knees to lower themselves, and arch their back to align with the eyepiece of the surgical microscope. The situation is even worse for the assistant as they must lean over the patient to look through the eyepiece, and it is often impossible to assist in these situations. In theory, an exoscope would allow surgeons to always operate in a neutral and ergonomically correct posture, regardless of the awkward and extreme angulations of the exoscope and tubular retractor, because visualizations are displayed on large digital monitors. Independence from the eyepiece allows the surgeon and assistant to work ergonomically and not contort their posture to see through the eyepiece.

Three-dimensional (3D) high definition (HD) monitors have been used for a long time in general surgery, obstetrics, arthroscopic work in orthopedics, and spinal endoscopy. 3D HD allows for superior task efficiency and reduces the procedural time and learning curve compared to two-dimensional (2D) HD monitors $[9,10]$. Advancements in digital monitors provide $4 \mathrm{~K}$ or ultrahigh definition, which has considerably higher resolution
$(3,840 \times 2,160$ pixels $)$ than HD monitors $(1,920 \times 1,080$ pixels). The increased resolution reduces pixilation especially when zooming, improving image sharpness, and clarity of extra fine details, which is particularly important when working around delicate and critical structures. Therefore, this technology enables surgeons to visualize the most intricate details clearly and with more contrast. Unfortunately, there have been no studies which have proven the effectiveness of $3 \mathrm{D} 4 \mathrm{~K}$ compared to the current standard of 3D HD medical grade monitors.

Intraoperative visualization and illumination are some of the key factors for successful spinal surgery. Loupes, microscopes, exoscopes, and endoscopes have been used for intraoperative visualization and illumination. The new Kinevo 900 (Carl Zeiss Meditec AG, Jena, Germany) integrates a microscope, an exoscope, and an endoscope (Qevo) into a single unit, allowing surgeons the freedom of choice regarding visualization during each step of surgery, as each mode of visualization has its own advantages and disadvantages. The microscope has long been regarded as the best tool for microsurgical visualization, but is limited by positioning at extreme angles, which compromises the surgeons neutral posture, which has detrimental long-term effects. An exoscope allows for excellent visualization at extreme angles as the surgeon no longer depends on the eyepiece but operates using the monitors. The main drawback of exoscopes is the limited depth perception, which is overcome using newer monitors like 3D or 3D $4 \mathrm{~K}$ monitors. Finally, the endoscope is best when used to visualize angles where the microscope or exoscope cannot reach, as the camera is positioned at an angle allowing for great visualization, such as during lateral recess decompression. Therefore, this new surgical microscope allows surgeons the choice of the traditional method of looking through eyepiece optics or an exoscope looking at three monitors that provide $2 \mathrm{D}, 3 \mathrm{D}$, and large $3 \mathrm{D} 4 \mathrm{~K}$ views of the surgical field. However, the value of solely depending on the exoscope and 3D $4 \mathrm{~K}$ monitors during microsurgical work remains to be proven.

The main objective of this prospective study is to evaluate the adaptation and learning curve when using an exoscope and 3D $4 \mathrm{~K}$ visualization in terms of operating time and blood loss. We assess the advantages, disadvantages, and surgical complications of exoscopes in tubular-access MISS. The secondary objective is to assess surgeon satisfaction with the exoscope and digital hybrid visualization during the surgery. 


\section{Materials and Methods}

This prospective case series was performed between Mac 2018 and January 2019 in a tertiary referral center. A convenient sampling method was used to recruit cases, and we collected data from all patients who underwent any form of decompression surgery using a tubular retractor and an exoscope equipped with a hybrid digital visualization system. During this period, 74 patients had such procedures. Five cases were excluded because of revision surgery (one revision decompression L5/ $\mathrm{S} 1$ and one revision for recurrent disc post endoscopic discectomy L4/5), infection (pyogenic discitis L4/5), and craniocervical junction transoral tubular decompression (myelopathy secondary to craniocervical junction stenosis from neglected os odontoideum and $\mathrm{C} 1 / \mathrm{C} 2$ dislocation in Larsen syndrome, with two cases). We documented the types of surgery (minimally invasive transforaminal lumbar interbody fusion [MIS TLIF], oblique lateral interbody fusion [OLIF], tubular decompression, or tubular microdiscectomy), operative time in minutes, blood loss in $\mathrm{mL}$, and complications that occurred during surgery. For multilevel cases, the average blood loss and operating time were used. In order to assess the learning curve and adaptation to the digital hybrid visualization, we divided the patients into the MIS TLIF group, tubular decompression group, tubular microdiscectomy group, and OLIF group. For each surgical procedure, we plotted sequential case series vs operative time to determine a plateau, which we interpreted as the proficiency or learning curve with the exoscope. The proficiency is defined once the learning curve reached a plateau on the plotted graph. Student $t$ test was used to determine significant differences in mean operating time before and after surgical proficiency was obtained. A $p$-value of $<0.05$ was statistically significant. Potential advantages were determined from surgeons' feedback, after they were asked regarding image quality and depth of perception, their ability to efficiently perform the target site treatment, and their ability to maintain good posture during the surgery. The residents' feedback regarding their ability to visualize and learn during surgery was also collected. The success of the surgery and clinical advantages were assessed by improvement in patient symptoms. The disadvantages assessed were the need to convert to open surgery or the need to use a microscope because of poor visualization or poor image quality. We also recorded complications during surgery, such as dural tear, excessive blood loss, postoperative neurological deficit, and postoperative surgical site infection. The institutional approval code for the study was TFF2019915. Informed consent was taken from all patients involved.

\section{Operating room, exoscope, and digital hybrid visual- ization monitors setup}

With the exoscope and digital hybrid visualization, there are few changes in the operating room which can streamline the work process.

\section{1) Position of the microscope/exoscope tower}

Contrary to a conventional microscope, which can be positioned behind or in front of the surgeon, conversion to digital visualization requires positioning of the digital hybrid visualization equipment in front of the surgeon, and the surgeon is located nearly perpendicular to the monitors for best 3D image visualization (Fig. 1A, B).

\section{2) Working distance}

The working distance of the exoscope is a massive 625 $\mathrm{mm}$, not only to clear the long instruments used in spine surgery but also for suspension well above the eyes of the surgeon so as not to block the surgeon's and assistant's line

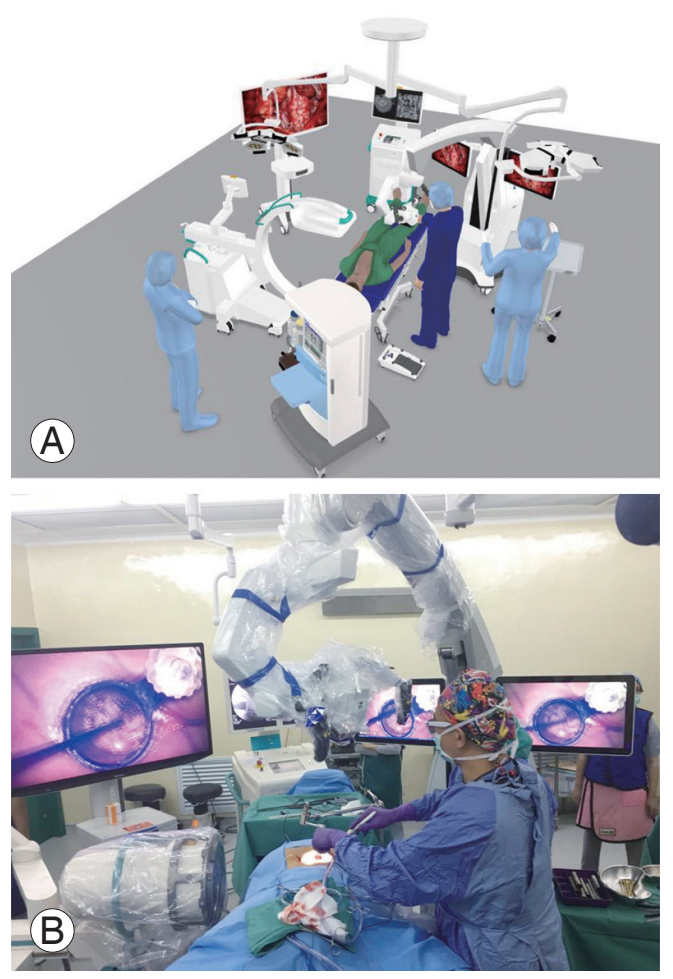

Fig. 1. (A, B) Intraoperative setup. 
Table 1. Summary of surgical procedure done

\begin{tabular}{|c|c|c|c|c|c|}
\hline Surgery & $\begin{array}{c}\text { No. of } \\
\text { patients }\end{array}$ & Level & $\begin{array}{l}\text { Average blood loss } \\
\text { (mL/level) }\end{array}$ & $\begin{array}{l}\text { Average operating } \\
\text { time (min/level) }\end{array}$ & Intraoperative complications \\
\hline Tubular decompression & 18 & 29 & 79.6 & 68 & 2 Dural tear (patient no. 5 and 13) \\
\hline Tubular discectomy & 17 & 19 & 91.1 & 62.9 & 2 Dural tear (patient no. 4 and 16) \\
\hline $\begin{array}{l}\text { Minimally invasive spine surgery } \\
\text { transforaminal lumbar inter- } \\
\text { body fusion }\end{array}$ & 28 & 32 & 155.8 & 88.9 & Nil \\
\hline Oblique lateral interbody fusion & 6 & 15 & 83.3 & 48.1 & Nil \\
\hline
\end{tabular}

of sight to the monitors. This massive working distance necessitates area clearance around the exoscope. In addition, as the system is also equipped with special features such as robotic repositioning and point lock, this further stresses the importance of keeping the area clear. This is done simply by moving the operating table/Jackson spine table away from the center, where usually the light pendants were placed.

\section{3) $\mathrm{C}$-arm/navigation and O-Arm}

MISS is dependent on the images provided by the C-arm and navigation. We always positioned them and simulated their predicted movement before the surgery to prevent collisions. This creates a streamlined approach to surgery and minimizes interruptions due to unforeseen equipment repositioning.

\section{4) 3D glasses}

Just like watching a 3D movie, the operating surgeon and surgical team wear $3 \mathrm{D}$ glasses. The nurses usually do not wear these glasses and will instead focus on the single 2D monitor. The 3D glasses have right and left circular polarized lens. The right and left eyes will then receive right or left image-specific signals which the brain will reprocess into a stereographic image, thus allowing better depth perception, which is vital in spine surgery. These glasses do not distort the normal view and are worn during the whole procedure, such as during percutaneous screw insertion, where 3D monitors are not used.

\section{Surgical technique}

The surgical technique used was similar to those performed using conventional surgical microscopes in our clinic since 2012. For microsurgical lumbar decompression and microdiscectomy, the extent of decompression, whether ipsilateral or both ipsilateral and contralateral, was dictated by patient symptoms and magnetic resonance imaging (MRI). For multilevel surgery, positioning the patient in lumbar lordosis allowed for a single incision to access up to three levels of decompression via the tubular retractor. Our MIS TLIF technique requires two incisions placed 0.5 to $1 \mathrm{~cm}$ lateral to the lateral border of the pedicle to be instrumented, allowing not only good medialization of the percutaneous screws but also an oblique central cage placement in the coronal plane. We believe in direct decompression; therefore, even with a cage inserted, we still performed ipsilateral and contralateral decompression as dictated by patient symptoms and MRI imaging. OLIF was done with the patient in a true lateral position. After skin marking, a muscle splitting approach is used before the tubular reactor is docked to perform discectomy and lateral inter body fusion.

\section{Results}

Out of 74 patients (99 levels), 69 patients (95 levels) were analyzed. The average operating time, the blood loss, and the intraoperative complications are shown in Table 1. From the graph (Figs. 2-4), the average operating time plateaued after six cases for tubular microdiscectomy and nine cases for tubular decompression and MIS TLIF. There was a significant difference in the mean operating time for MIS TLIF, tubular decompression, and tubular discectomy (Table 2). We did not perform enough OLIF cases to accurately determine a plateau time.

All three surgeons agreed that the image quality and depth perception were excellent for target site treatment. They also gave positive feedback regarding their ability to maintain neutral and ergonomic posture throughout the procedure. Residents also reported a positive learning experience in the operating theater from better visualization and depth perception. Two trainees also reported experiencing a headache when wearing the $3 \mathrm{D}$ glasses. However, none of 


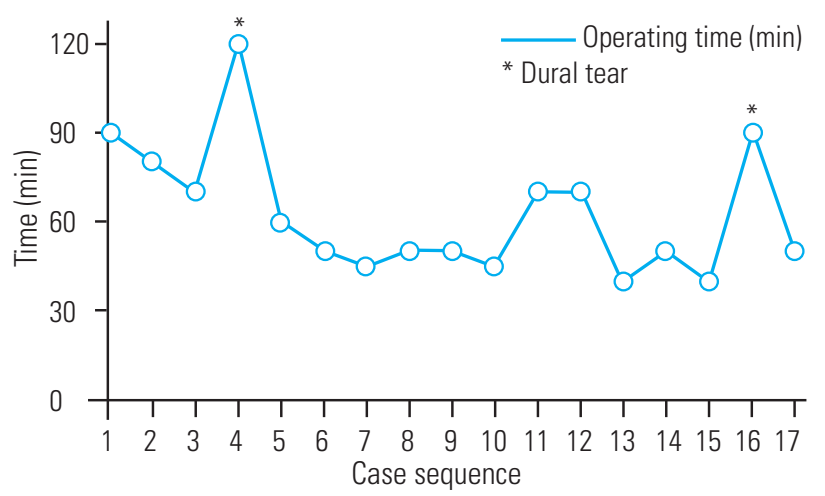

Fig. 2. Learning curve tubular microdiscectomy.

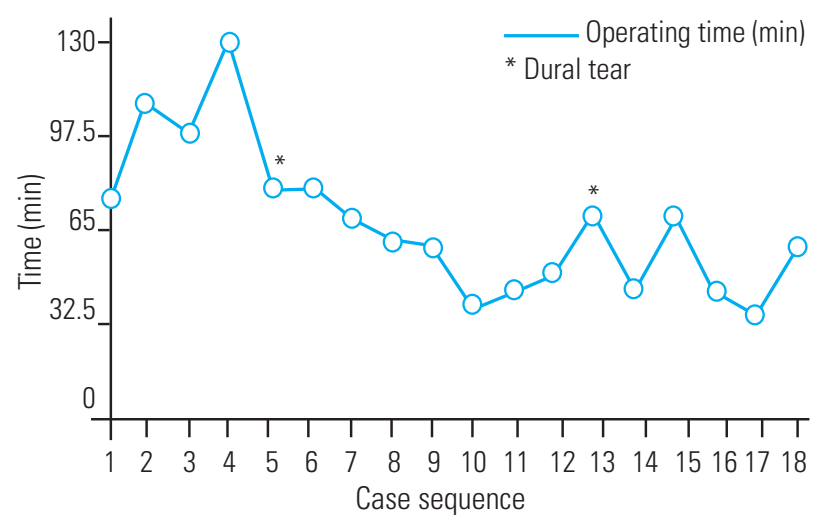

Fig. 3. Learning curve tubular decompression.

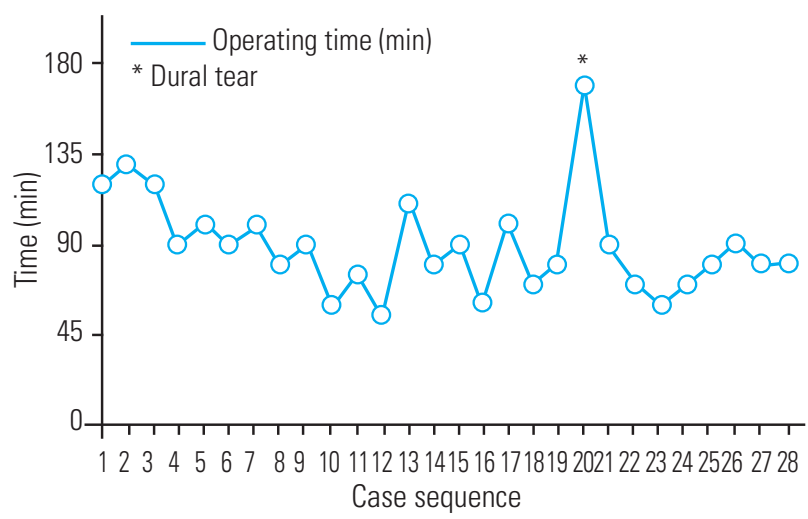

Fig. 4. Learning curve minimally invasive spine surgery transforaminal lumbar interbody fusion. the surgeons experienced such headache symptoms.

All patients experienced symptomatic improvement for radicular pain and neurological function post operatively, and there were no postoperative neurological deficits. No case requires conversion to open surgery or change of exoscope to microscope because of poor visualization or image quality. As for intraoperative complications, we had four cases of dural tear (case sequence numbers 20, 21,52 , and 71 ), which were all managed using surgical sealant and 24 hours of bed rest, and no cases experienced postoperative headache or leak. We also had one case of surgical site infection after a tubular microdiscectomy that required a second surgery 18 days after index surgery, during which we found that the infection was actually superficial.

\section{Discussion}

The concern about exoscopes involved not only adaptation and learning curves but also concerns about trusting the digital image quality compared to traditional eyepiece optics. In this study, all three surgeons unanimously agreed that hybrid digital visualization provides excellent visualization for successful treatment at the target site, as determined by symptomatic improvement in patient radiculopathy and neurological function. These data suggest that the exoscope with a hybrid visualization system offers excellent visualization, high contrast, excellent depth perception, and clarity for precision target site treatment and successful surgical and clinical outcomes. Residents gave positive feedback on image quality, which helped their learning ability during surgery. Khalessi et al. [11] previously reported excellent optics in their review of $18 \mathrm{mi}$ croneurosurgery cases operated using a 3D HD exoscope.

Our study also showed that the learning curve and adaptation to the exoscope are rather short. This is new information as no other studies on exoscope for cranial

Table 2. Mean operating time before and after adaptation into exoscope

\begin{tabular}{lccc} 
Type of surgery & $\begin{array}{c}\text { Mean operating time } \\
\text { before adaptation (min) }\end{array}$ & $\begin{array}{c}\text { Mean operating time after } \\
\text { adaptation (min) }\end{array}$ & $\begin{array}{c}p \text {-value } \\
\text { Tubular decompression }\end{array}$ \\
\hline $\begin{array}{l}\text { Tubular discectomy } \\
\text { Minimally invasive spine surgery transforaminal } \\
\text { lumbar interbody fusion }\end{array}$ & $78.0 \pm 23.7$ & $51.1 \pm 12.7$ & 0.002 \\
\hline
\end{tabular}

Values are presented as mean \pm standard deviation. 
work report on the learning curve. In a retrospective review involving 10 patients using an HD-3D exoscope for spine surgery, Kwan et al. [12] experienced excellent surgical and clinical outcomes without any complications, concluding that the exoscope is feasible for spinal surgery.

With the exoscope, surgeons maintain good posture throughout surgery, which is advantageous for well-being and longevity. Better ergonomics of the spine during surgery is desired by all spine surgeons because of long working hours and strain on the neck, shoulder, arms, and lower back, especially during contralateral decompression procedures when the microscope is positioned at extreme working angles. Using an exoscope, surgeons will be able to independently position themselves independent of the exoscope position as they now focus on the digital hybrid visualization system rather than being encumbered by the oculars (Fig. 5). Better ergonomics also extends to the assistant and the entire surgical team. This ergonomic advantage and reduced surgeon fatigue from a fixed unnatural posture were also highlighted by Khalessi et al. [11] and Garneau et al. [13].

The complications which occurred in our study are comparable to those using the surgical microscope. Although we did not observe any disadvantages, reduced depth perception has previously been reported [13]. The only drawback of using an exoscope is the need to rearrange the operating theater setup as the monitors must be placed in front of the surgeons, which initially disturbs the usual arrangements and space management in the operating theater. The higher working distance of the exoscope also necessitates that the area around the exoscope is kept clear. To overcome this problem, we performed a

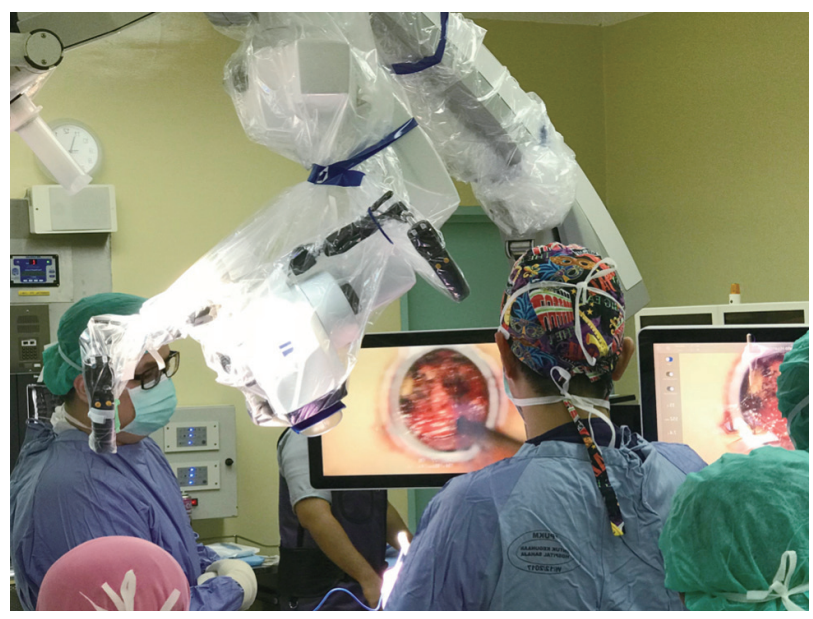

Fig. 5. Surgeon being independent of the exoscope. few simulations in the operating theater prior to surgery and determined a new operating theater layout based on the type of surgery performed to streamline the work process. With further improvement in digital technology, we hope that future advances obviate the need for 3D glasses. Finally, although this is the largest case series to examine using an exoscope and digital hybrid visualization, future studies should quantify image quality, student satisfaction, and surgeon satisfaction in a more objective manner.

\section{Conclusions}

The exoscope with hybrid digital visualization provides excellent visualization, depth perception, and clarity for successful target site treatment. It allows surgeons and assistants to operate with better ergonomics, avoiding stress and fatigue from uncomfortable body positions. Residents also enjoy better operative teaching. The only drawback is the need to rearrange the operating theater layout and setup. The learning curve is rather short, and potential complications are comparable to using a surgical microscope.

\section{Conflict of Interest}

No potential conflict of interest relevant to this article was reported.

\section{References}

1. Williams RW. Microlumbar discectomy: a conservative surgical approach to the virgin herniated lumbar disc. Spine (Phila Pa 1976) 1978;3:175-82.

2. Goald HJ. Microlumbar discectomy: followup of 147 patients. Spine (Phila Pa 1976) 1978;3:183-5.

3. Caspar W, Campbell B, Barbier DD, Kretschmmer R, Gotfried Y. The Caspar microsurgical discectomy and comparison with a conventional standard lumbar disc procedure. Neurosurgery 1991;28:78-86.

4. Overdevest GM, Peul WC, Brand R, et al. Tubular discectomy versus conventional microdiscectomy for the treatment of lumbar disc herniation: long-term results of a randomised controlled trial. J Neurol Neurosurg Psychiatry 2017;88:1008-16.

5. Gempt J, Jonek M, Ringel F, Preuss A, Wolf P, Ryang Y. Long-term follow-up of standard microdiscectomy versus minimal access surgery for lumbar disc her- 
niations. Acta Neurochir (Wien) 2013;155:2333-8.

6. Brock M, Kunkel P, Papavero L. Lumbar microdiscectomy: subperiosteal versus transmuscular approach and influence on the early postoperative analgesic consumption. Eur Spine J 2008;17:518-22.

7. Schick U, Dohnert J, Richter A, Konig A, Vitzthum HE. Microendoscopic lumbar discectomy versus open surgery: an intraoperative EMG study. Eur Spine J 2002;11:20-6.

8. Kawaguchi Y, Matsui H, Tsuji H. Back muscle injury after posterior lumbar spine surgery: a histologic and enzymatic analysis. Spine (Phila Pa 1976) 1996;21:941-4.

9. Storz P, Buess GF, Kunert W, Kirschniak A. 3D HD versus 2D HD: surgical task efficiency in standardised phantom tasks. Surg Endosc 2012;26:145460 .
10. Guana R, Ferrero L, Garofalo S, et al. Skills comparison in pediatric residents using a 2-dimensional versus a 3-dimensional high-definition camera in a pediatric laparoscopic simulator. J Surg Educ 2017;74:644-9.

11. Khalessi AA, Rahme R, Rennert RC, et al. First-inman clinical experience using a high-definition 3-dimensional exoscope system for microneurosurgery. Oper Neurosurg (Hagerstown) 2019;16:717-25.

12. Kwan K, Schneider JR, Du V, et al. Lessons learned using a high-definition 3-dimensional exoscope for spinal surgery. Oper Neurosurg (Hagerstown) 2019;16:619-25.

13. Garneau JC, Laitman BM, Cosetti MK, Hadjipanayis C, Wanna G. The use of the exoscope in lateral skull base surgery: advantages and limitations. Otol Neurotol 2019;40:236-40. 